\title{
Epithelioid Sarcoma in the Cervical Spine: A Case Report
}

\author{
Chungnam Lee ${ }^{1}$, Woo Jin Choe', Nara Kim² \\ Departments of ${ }^{I}$ Neurosurgery and ${ }^{2}$ Radiology, Konkuk University College of Medicine, Seoul, Korea
}

Epithelioid sarcoma is a rare and highly malignant soft tissue neoplasm that most commonly occurs in the long bones. This uncommon tumor has a poor clinical outcome, and the modality of its treatment has not yet been fully established. The authors report an extremely rare presentation of epithelioid sarcoma in the cervical spine, along with its clinical progression, imaging, and pathology. The patient underwent three surgical procedures and adjuvant radiochemical management. He survived for 25 months with a good general condition and adapted well to his social activity. Systemic metastasis was not found, but the patient died of respiratory failure due to direct tracheal invasion of the tumor.

Key Words: Epithelioid sarcoma $\cdot$ Cervical spine $\cdot$ Chemotherapy

\section{INTRODUCTION}

Epithelioid sarcoma is an uncommon soft tissue tumor that accounts for less than $1 \%$ of all soft tissue sarcomas found in young adults. It is found more frequently in men than in women $^{3,4)}$. The tumor has been reported in various locations in the body, including the head, neck, chest wall, abdominal wall, genital region, and extremities. Epithelioid sarcoma mostly involves the subcutaneous tissue, tendons, and fascia. It is commonly located in the extremities, and rarely occurs in the axial skeleton", especially in the spine, where it has been reported in the literature in less than 10 cases and in only one case in the cervical spine ${ }^{2)}$. Due to its rareness and aggressive features, its treatment modality has not yet been established. The authors report an extremely rare presentation of epithelioid sarcoma in the cervical spine and its clinical course.

\section{CASE REPORT}

A 19-year-old man complained right shoulder pain for three weeks. A week before visit to hospital, he experienced bilateral upper extremity weakness that eventually spread to his lower

- Received: June 24, 2015 • Revised: September 4, 2015

- Accepted: September 7, 2015

Corresponding Author: Woo Jin Choe, MD, PhD

Department of Neurosurgery, Konkuk University Medical Center, Konkuk University College of Medicine, 120-1 Neungdong-ro, Gwangjin-gu,

Seoul 05030, Korea

Tel: +82-2-2030-7357, Fax: +82-2-2030-7359

E-mail:20050100@kuh.ac.kr

@This is an Open Access article distributed under the terms of the Creative Commons Attribution Non-Commercial License (http://creativecommons.org/ licenses/by-nc/3.0/) which permits unrestricted non-commercial use, distribution, and reproduction in any medium, provided the original work is properly cited. extremities and resulted in his admission to our hospital via the emergency room. At the time of his admission, his neurological exam revealed quadriparesis, with a motor grade of grade 2 out of 5 for his right upper extremity (UE), grade 3 for his left upper extremity, and grade 4 for his right lower extremity (LE). He exhibited hypesthesia at his UE/LE and an increased deep tendon response at his LE.

The magnetic resonance imaging (MRI) of his cervical spine showed a huge lobulating mass at the right $\mathrm{C} 3-\mathrm{C} 7$ level with an invasion of C5-6 neural foramen. The tumor caused bone erosion in the C4-5 vertebral body. Moreover, the tumor extended into the central canal of the C3-5 level, which caused spinal cord myelopathy. The lesion had relatively low signal intensity on the T2-weighted images and isointense on the T1-weighted images analogous to the muscle signal intensity. In the post-contrast image, the tumor was well-enhanced with heterogeneous signals (Fig. 1).

We performed transfemoral spinal angiography and tumor embolization before surgical treatment. The patient underwent resection of the tumor through the combined anteriorposterior approach. First, to decompress the myelopathy, we used a posterior approach. Epidural mass decompressive tumorectomy through the posterior approach. We performed a C3 to $\mathrm{C} 6$ posterior fusion. And second stage operation was performed by subtotal resection of the epidural tumor through the anterolateral approach. The tumor was whitish with a firm mass and was highly vascularized. The adhesion of tumor to the surrounding tissues was moderate. The tumor had enclosed vertebral artery and nerve root. The pathological diagnosis was epithelioid sarcoma. The immune-histochemical study results were positive for beta-catenin, CD99, epithelial membrane antigen (EMA), and vimentin. The negative S-100 staining excluded the diagnosis of a malignant peripheral nerve sheath 

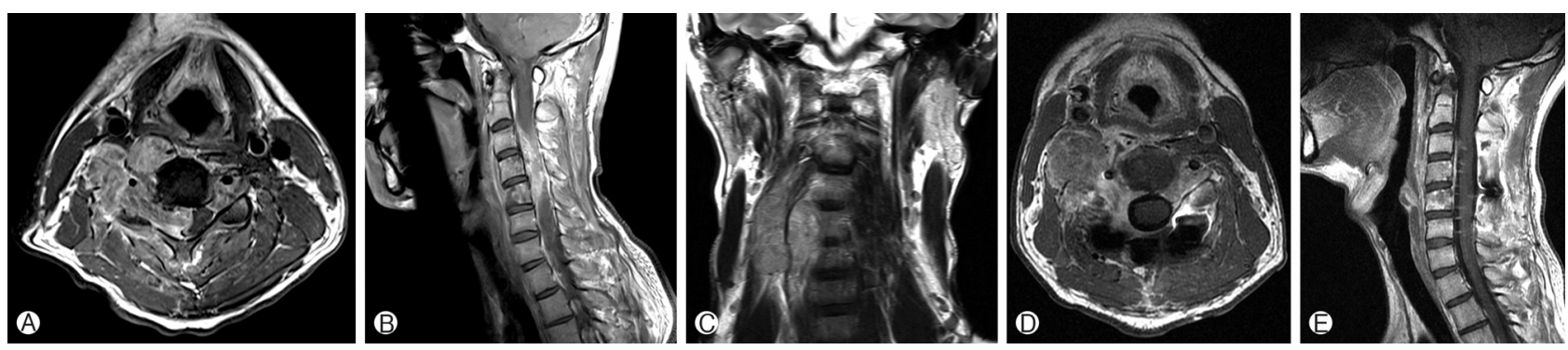

Fig. 1. T1-weighted MRI in the axial (A), sagittal (B), and T2 MRI coronal (C) of the cervical spine: The MRI of the cervical spine revealed a huge heterogenous paravertebral mass on the right side. The mass was extended through central canal from C3 to C5. And there was daughter mass lesion with compression of C4-5 neural foramen. Postoperative sagittal MRI in the axial (D), sagittal (E): The MRI shows that the tumor is removed as much as possible and there is no evidence of instability and cord compression.

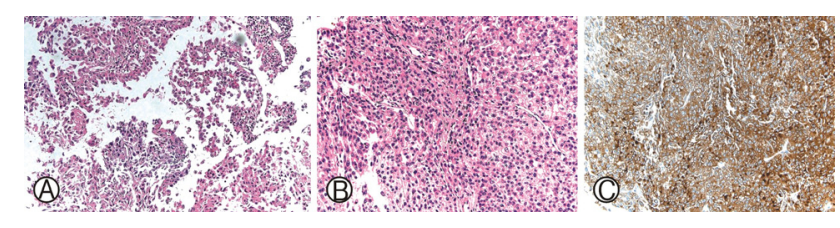

Fig. 2. Histopathologic aspect of epithelioid sarcoma. Histological specimens from the spinal mass show spindle cells. The tumor cells are roundish, admixed with spindle shaped cells with featuring an eosinophilic cytoplasm. (A: H\&E $\times 100$ and B: H\&E $\times 200$ ). (C) Tumor cells expressing vimentin: The result of the immunohistochemical study is positive for vimentin and conspicuous atypical mitotic activity.

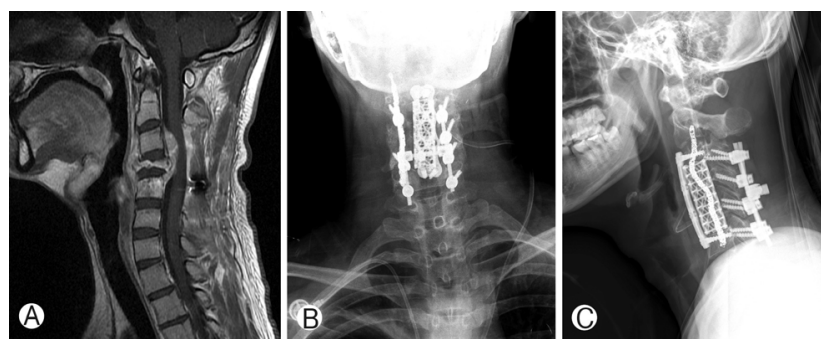

Fig. 3. (A) Follow-up MRI of the cervical spine showed a newly pathologic compression fracture at C4 that involved large epidural mass formation. Post-operative plain radiograph of the cervical spine at the final follow-up: (B) anteroposterior and (C) lateral.

tumor (Fig. 2). Postoperatively, the patient's symptoms such as weakness and hypesthesia improved gradually. Three weeks after surgical treatment, primary chemotherapy with ifosamide and adriamycin was performed. The patient showed no neurological change, but follow-up MRI after four months showed a newly pathologic compression fracture at $\mathrm{C} 4$ that involved large epidural mass formation. The patient underwent third operation that included C4-5 corpectomy and fusion from C3 to $\mathrm{C6}$ (Fig. 3). After last surgical treatment, chemotherapy regimen was changed to gemcitabine/docetaxel, followed by adjuvant radiation therapy. The total dose administered was 4,000 cGy in 30 fractions over 4 weeks. The patient received five courses of chemotherapy in 12 weeks. He was initially stable, but he underwent acute respiratory distress. He died after having survived for 25 months after diagnosis.

\section{DISCUSSION}

Epithelioid sarcoma is rare, accounting for $<1 \%$ of cases of soft tissue sarcoma with aggressive mesenchymal features of unknown histogenesis, and displays multidirectional differentiation, which comprises predominantly epithelial cells. Microscopic involvement of the skin and the skeletal muscle has been seen in $24 \%$ and $28 \%$ of cases, respectively. The usual gross appearance of epithelioid sarcoma consists of one or more white nodules with infiltrating margins. It was first described in 1961 by Lakowski as "sarcoma aponeuroticum," and was renamed as "epithelioid sarcoma" by Enzinger in $1970^{4,10,13,15}$. Epithelioid sarcoma in the spine was reported in only 8 cases until 2013, and a cervical spine case was first described in $2013^{2)}$. Our case is the ninth reported case in the entire spine and the second reported case in the cervical spine (Table 1).

The histological characteristics of epithelioid sarcoma are similar to those of inflammatory processes and other benign soft tissue tumors. Histopathologically, epithelioid sarcoma has many analogous patterns, such as granuloma annulare, necrobiosis lipoidica, and chronic granulomatous inflammation ${ }^{2)}$. Its histological characteristics resemble those of various benign and malignant conditions, including chronic granulomatous inflammation, nodular fasciitis, and synovial sarcoma ${ }^{2}$. The tumor consists of epithelial-appearing (ovoid or polygonal) cells that are well-blended with fusiform cells, which are strongly eosinophilic, with many containing intracytoplasmic vacuoles ${ }^{1}$. The most common pattern seen is the "pseudo-granulomatous" proliferation of cells around an acellular, necrotic central zone $^{13)}$. Differential diagnosis of epithelioid sarcoma from other forms of cancer is required through various immunohistoche- 
Table 1. Characteristics of spinal epithelioid sarcoma

\begin{tabular}{|c|c|c|c|c|c|c|c|}
\hline Author, year & Age & Sex & Location & Necrosis & "Spinal canal extension & Therapy & Survival \\
\hline Steib et al., $1996^{11)}$ & 35 & $M$ & Sacrum & $Y$ & $Y$ & $\mathrm{~S}, \mathrm{C}, \mathrm{RT}$ & 3 \\
\hline Tournigand et al., 1997 & 23 & $M$ & Coccyx & N/A & N/A & $\mathrm{S}, \mathrm{C}, \mathrm{RT}$ & 9 \\
\hline Ilaslan et al., $2004^{8)}$ & $\mathrm{N} / \mathrm{A}$ & $\mathrm{N} / \mathrm{A}$ & N/A & N/A & $\mathrm{N} / \mathrm{A}$ & $\mathrm{N} / \mathrm{A}$ & N/A \\
\hline Weisskopf et al., 2006 $6^{(4)}$ & 14 & M & Thoracic & N & Y & $\mathrm{S}, \mathrm{C}, \mathrm{RT}$ & 37 \\
\hline Chen et al., 2007 & 5 & $\mathrm{~F}$ & Sacrum & $\mathrm{N} / \mathrm{A}$ & N/A & $\mathrm{N} / \mathrm{A}$ & N/A \\
\hline Chanplakorn et al., $2011^{5)}$ & 14 & M & Thoracic & Y & Y & $\mathrm{S}, \mathrm{C}, \mathrm{RT}$ & 40 \\
\hline Yoo et al., $2011^{16)}$ & 29 & M & Lumbar & Y & Y & $\mathrm{S}, \mathrm{C}, \mathrm{RT}$ & N/A \\
\hline Babu $R$ et al., 2013 & 25 & $\mathrm{~F}$ & Cervical & Y & Y & S & N/A \\
\hline Current report & 19 & M & Cenvical & Y & Y & $\mathrm{S}, \mathrm{C}, \mathrm{RT}$ & 25 \\
\hline
\end{tabular}

mical stains such as CD99, EMA, vimentin, and $\mathrm{S}-100^{2,7)}$.

The treatment of choice is wide resection and chemoradiotherapy ${ }^{9,14)}$. Despite multimodal treatment, this type of tumor has a poor clinical outcome and a high rate of local recurrence, and its treatment modality has not yet been fully established ${ }^{2)}$.

The case reported herein is an extremely rare presentation of epithelioid sarcoma in the cervical spine. For decompression of the spinal cord, we had planned to perform surgical treatment. The transfemoral spinal angiography showed that the tumor had a large hypervascularity in the right hemiverbetral/paraspinal area at the C3-4-5 levels. We performed preoperative embolization of the tumor and segmental occlusion of the vertebral artery. This procedure effectively controlled the bleeding during the surgery and prevented vertebral artery rupture. The patient's myelopathy improved after immediate post-operation, so he started chemotherapy with ifosamide/ adriamycin. Four months after surgery, MRI showed a newly pathologic compression fracture at $\mathrm{C} 4$ with new epidural mass formation (Fig. 3). He underwent a third operation with tumorectomy and corpectomy at C4 and C5. Through an anterior approach, the safety of the nerve root and the vertebral artery was confirmed. The chemotherapy treatment regimen was changed from ifosamide/adriamycin to gemcitabine/docetaxel for an aggressive recurrent tumor. According to the literature of thoracic spine cases, the patient was administered quadruple chemotherapy agents (ifosfamid, vincristin, actinomycin, and doxorubicin) ${ }^{14)}$. In our case, he had a good general condition and adapted well to social activities. He was on maintenance medication until just prior to his death, without deterioration in his reported weakness. The patient died due to respiratory arrest after 25 months from the first surgical treatment. When he visited the outpatient care before death, there was regrowth of the mass, and he complained of dyspnea due to airway compression. We recommended surgery, but his parents refused.

Compared to previously reported cases, the patient in our case was thought to have had good disease control. A spinal epithelioid sarcoma case in 1996 reported that the patients had up to three months of post-operative mortality ${ }^{11)}$, while a case reported in 1997 had up to nine months ${ }^{12)}$. Our case differed from these two cases in that we actively changed the chemotherapy after the recurrent tumor was subjected to radiotherapy and surgery. The prognosis of epithelioid sarcoma is very poor, but multimodal treatment is clearly important to raise the average survival rate of patients.

\section{CONCLUSION}

Epithelioid sarcoma in the spine is extremely rare, and initial radical wide resection in addition to chemoradiotherapy is beneficial. However, further studies and the accumulation of cases are needed to investigate unknown factors related to spinal epithelioid sarcoma.

\section{REFERENCES}

1. Armah HB, Parwani AV: Epithelioid sarcoma. Arch Pathol Lab Med 133:814-819, 2009

2. Babu R, Karikari IO, Cummings TJ, Gottfried ON, Bagley CA: Treatment and outcomes of epithelioid sarcoma of the spine. J Clin Neurosci 20:1342-1345, 2013

3. Baratti D, Pennacchioli E, Casali PG, Bertulli R, Lozza L, Olmi P, et al: Epithelioid sarcoma: Prognostic factors and survival in a series of patients treated at a single institution. Ann Surg Oncol 14:3542-3551, 2007

4. Bos GD, Pritchard DJ, Reiman H, Dobyns J, Ilstrup D, Landon G: Epithelioid sarcoma. An analysis of fifty-one cases. J Bone Joint Surg Am 70:862-870, 1988

5. Chanplakorn P, Chanplakorn N, Pongtippan A, Jaovisidha S, Laohacharoensombat W: Recurrent epithelioid sarcoma in the thoracic spine successfully treated with multilevel total en bloc spondylectomy. Eur Spine J 20:S302-308, 2011 
6. Chen YW, Huang MY, Chang CC, Lee CS, Liao YM, Chiu SS, et al: FDG PET/CT findings of epithelioid sarcoma in a pediatric patient. Clin Nucl Med 32:898-901, 2007

7. Eyden B, Wang G, Yao L: Epithelioid sarcoma: a case report with ultrastructural confirmation of myofibroblastic differentiation based on fibronexus junctions. Ultrastruct Pathol 33:61-66, 2009

8. Ilaslan H, Sundaram M, Unni KK, Shives TC: Primary vertebral osteosarcoma: imaging findings. Radiology 230:697-702, 2004

9. Kneisl JS, Coleman MM, Raut CP: Outcomes in the management of adult soft tissue sarcomas. J Surg Oncol 110:527-538, 2014

10. Rodríguez NG, Cancelo ÁP, Ruán JI, Pérez MG: Epithelioid sarcoma of the right ilium mimicking sacroiliitis. Reumatol Clín 9:120-122, 2013

11. Steib JP, Pierchon F, Farcy JP, Lang G, Christmann D, Gnassia JP: Epithelioid sarcoma of the spine: A case report. Spine (Phila
Pa 1976) 21:634-638, 1996

12. Tournigand C, Bernard O, Cruel T, Sarrazin JL, Merrer J: Epithelioid sarcoma. Diagnostic and therapeutic aspects apropos of a coccygeal localization. Rev Med Interne 18:50-53, 1997

13. Wadhwa V, Salaria SN, Thakkar RS, Chhabra A: Epithelioid sarcoma presenting as radial mononeuropathy: anatomical, magnetic resonance neurography and diffusion tensor imaging appearances. Skeletal Radiol 42:853-858, 2013

14. Weisskopf M, M?nker R, Hermanns-Sachweh B, Ohnsorge JA, Siebert C: Epithelioid sarcoma in the thoracic spine. Eur Spine J 15:604-609, 2006

15. Yamato M, Nishimura G, Yamaguchi T, Tamai K, Saotome K: Epithelioid sarcoma with unusual radiological findings. Skeletal Radiol 26:606-610, 1997

16. Yoo JH: Epithelioid sarcoma of the spine: A case report and review. Open J Clin Diagn 1:1-4, 2011 